\title{
Common fixed point theorems for weakly increasing mappings on ordered uniform spaces
}

\author{
Ishak Altun
}




\title{
COMMON FIXED POINT THEOREMS FOR WEAKLY INCREASING MAPPINGS ON ORDERED UNIFORM SPACES
}

\author{
ISHAK ALTUN
}

Received 28 April, 2010

\begin{abstract}
In the present paper, we give a common fixed point theorem for two weakly increasing mappings on ordered Hausdorff uniform spaces, using the distance $p$. Two multivalued versions of this result are also presented.
\end{abstract}

2000 Mathematics Subject Classification: Primary 54H25; Secondary 47H10

Keywords: fixed point, uniform space, order relation

\section{INTRODUCTION}

Recently, Aamri and El Moutawakil [2] have introduced the concept of $E$-distance function on uniform spaces and utilized it to improve some well known results of the existing literature involving both $E$-contractive or $E$-expansive mappings. In [3], a partial ordering on uniform spaces has been introduced utilizing $E$-distance function and some fixed point theorems for single valued and multivalued nondecreasing (not necessary $E$-contractive or $E$-expansive) mappings on ordered uniform spaces have been proved. In this paper, we give a common fixed point theorem for two weakly increasing single valued mappings, which may not be nondecreasing, on ordered uniform spaces. Two multivalued versions of this result are also given.

In order to have a possibly self contained presentation, we recall some relevant definitions and properties of the foundation of uniform spaces. For further informations and details, one is referred to the book by Bourbaki [5]. We call a pair $(X, \vartheta)$ to be a uniform space if it consists of a non-empty set $X$ together with an uniformity $\vartheta$, where we assume that the latter begins with a special kind of filter on $X \times X$ whose all elements contain the diagonal $\Delta=\{(x, x): x \in X\}$. If $V \in \vartheta$ and $(x, y) \in V$, $(y, x) \in V$ then $x$ and $y$ are said to be $V$-close. A sequence $\left\{x_{n}\right\}$ in $X$, is said to be a Cauchy sequence w.r.t. the uniformity $\vartheta$ if for any $V \in \vartheta$, there exists $N \geq 1$ such that $x_{n}$ and $x_{m}$ are $V$-close for $m, n \geq N$. An uniformity $\vartheta$ defines a unique topology $\tau(\vartheta)$ on $X$ for which the neighborhoods of $x \in X$ are the sets $V(x)=\{y \in X:(x, y) \in V\}$ when $V$ runs over $\vartheta$. 
A uniform space $(X, \vartheta)$ is said to be Hausdorff if and only if the intersection of all the $V \in \vartheta$ reduces to the diagonal $\Delta$ of $X$, i.e. $(x, y) \in V$ for all $V \in \vartheta$ implies $x=y$. Notice that Hausdorffness of the topology induced by the uniformity guarantees the uniqueness of limit of a sequence in uniform spaces. An element of uniformity $\vartheta$ is said to be symmetrical if $V=V^{-1}=\{(y, x):(x, y) \in V\}$. Since each $V \in \vartheta$ contains a symmetrical $W \in \vartheta$ and if $(x, y) \in W$ then $x$ and $y$ are both $W$ and $V$-close, so one may assume that each $V \in \vartheta$ is symmetrical. When topological concepts are mentioned in the context of a uniform space $(X, \vartheta)$, they are naturally interpreted with respect to the topological space $(X, \tau(\vartheta))$.

\section{PReliminaries}

We shall use the following definitions and lemmas in the sequel.

Definition 1 ([2]). Let $(X, \vartheta)$ be a uniform space. A function $p: X \times X \rightarrow \mathbb{R}^{+}$is said to be an $E$-distance if

$\left(\mathrm{p}_{1}\right)$ For any $V \in \vartheta$ there exists $\delta>0$ such that $p(z, x) \leq \delta$ and $p(z, y) \leq \delta$, for some $z \in X$, imply $(x, y) \in V$,

$\left(\mathrm{p}_{2}\right) p(x, y) \leq p(x, z)+p(z, y), \forall x, y, z \in X$.

One can find some examples of $E$-distances in [2]. Especially, one can infer from Example 1 (contained in [2]) that every metric $d$ on a uniform space $(X, \vartheta)$ is an $E$-distance.

The following lemma embodies some useful properties of $E$-distances.

Lemma 1 ([1], [2]). Let $(X, \vartheta)$ be a Hausdorff uniform space and $p$ be an Edistance on $X$. Let $\left\{x_{n}\right\}$ and $\left\{y_{n}\right\}$ be arbitrary sequences in $X$ and $\left\{\alpha_{n}\right\},\left\{\beta_{n}\right\}$ be sequences in $\mathbb{R}^{+}$converging to 0 . Then, for $x, y, z \in X$, the following holds:

(a) If $p\left(x_{n}, y\right) \leq \alpha_{n}$ and $p\left(x_{n}, z\right) \leq \beta_{n}$ for all $n \in \mathbb{N}$, then $y=z$. In particular, if $p(x, y)=0$ and $p(x, z)=0$, then $y=z$.

(b) If $p\left(x_{n}, y_{n}\right) \leq \alpha_{n}$ and $p\left(x_{n}, z\right) \leq \beta_{n}$ for all $n \in \mathbb{N}$, then $\left\{y_{n}\right\}$ converges to $z$.

(c) If $p\left(x_{n}, x_{m}\right) \leq \alpha_{n}$ for all $m>n$, then $\left\{x_{n}\right\}$ is a Cauchy sequence in $(X, \vartheta)$.

Let $(X, \vartheta)$ be a uniform space equipped with $E$-distance $p$. A sequence in $X$ is $p$-Cauchy if it satisfies the usual metric condition. There are several concepts of completeness in this setting.

Definition 2 ([1], [2]). Let $(X, \vartheta)$ be a uniform space and $p$ be an $E$-distance on $X$. Then

(i) $X$ said to be $S$-complete if for every $p$-Cauchy sequence $\left\{x_{n}\right\}$ there exists $x \in X$ with $\lim _{n \rightarrow \infty} p\left(x_{n}, x\right)=0$,

(ii) $X$ is said to be $p$-Cauchy complete if for every $p$-Cauchy sequence $\left\{x_{n}\right\}$ there exists $x \in X$ such that $\lim _{n \rightarrow \infty} x_{n}=x$ with respect to $\tau(\vartheta)$, 
(iii) $f: X \rightarrow X$ is $p$-continuous if $\lim _{n \rightarrow \infty} p\left(x_{n}, x\right)=0$ implies

$$
\lim _{n \rightarrow \infty} p\left(f x_{n}, f x\right)=0
$$

and

(iv) $f: X \rightarrow X$ is $\tau(\vartheta)$-continuous if $\lim _{n \rightarrow \infty} x_{n}=x$ with respect to $\tau(\vartheta)$ implies $\lim _{n \rightarrow \infty} f x_{n}=f x$ with respect to $\tau(\vartheta)$.

Remark 1 ([2]). Let $(X, \vartheta)$ be a Hausdorff uniform space and let $\left\{x_{n}\right\}$ be a $p$ Cauchy sequence. Suppose that $X$ is $S$-complete, then there exists $x \in X$ such that $\lim _{n \rightarrow \infty} p\left(x_{n}, x\right)=0$. Then Lemma 1 (b) gives that $\lim _{n \rightarrow \infty} x_{n}=x$ with respect to the topology $\tau(\vartheta)$. This shows that $S$-completeness implies $p$-Cauchy completeness.

Using the following lemma, whose metric version is available in [6], we can construct an order relation on a Hausdorff uniform space.

Lemma 2 ([3]). Let $(X, \vartheta)$ be a Hausdorff uniform space, $p$ be E-distance on $X$ and $\phi: X \rightarrow \mathbb{R}$. Define the relation $\preceq$ on $X$ as follows:

$$
x \preceq y \Longleftrightarrow x=y \text { or } p(x, y) \leq \phi(x)-\phi(y) .
$$

Then $\preceq$ is a (partial) order on $X$ induced by $\phi$.

There are some examples in [3] for partial orders on a Hausdorff uniform space induced by $\phi$.

The following theorems have been given in [3].

Theorem 1. Let $(X, \vartheta)$ be a Hausdorff uniform space and $p$ be an E-distance on $X$ such that $X$ is $S$-complete and $\phi: X \rightarrow \mathbb{R}$ be a function which is from bounded from below. If $\preceq$ is the partial order induced by $\phi$ and $f: X \rightarrow X$ is a $p$-continuous or $\tau(\vartheta)$-continuous non-decreasing function with $x_{0} \preceq f x_{0}$ for some $x_{0} \in X$, then $f$ has a fixed point in $X$.

Theorem 2. Let $(X, \vartheta)$ be a Hausdorff uniform space equipped with an E-distance $p$ such that $X$ is $S$-complete and $\phi: X \rightarrow \mathbb{R}$ is bounded from below. Assume that $\preceq$ is the partial order induced by $\phi$. Then it follows that if $F: X \rightarrow 2^{X}$ is $p$-order closed operator with $\left\{x_{0}\right\} \prec_{1} F x_{0}$ for some $x_{0} \in X$ and for all $x, y \in X$, $x \preceq y \Longrightarrow F x \prec_{1} F y$ (i.e. $F$ is non-decreasing with respect to $\prec_{1}$ ), then $F$ has a fixed point in $X$.

The aim of this paper is to give some common fixed point theorems for single valued and multivalued weakly increasing mappings on ordered Hausdorff uniform spaces.

First, we recall the concept of weakly increasing mappings.

Definition 3 ([7], [8]). Let $(X, \preceq)$ be a partially ordered set. Two mappings $f, g$ : $X \rightarrow X$ are said to be weakly increasing if $f x \preceq g f x$ and $g x \preceq f g x$ hold for all $x \in X$. 
Note that two weakly increasing mappings need not be nondecreasing.

Example 1 ([4]). Let $X=\mathbb{R}^{2}$ be endowed with lexicographical ordering, that is, $(x, y) \preceq(z, w) \Leftrightarrow(x<z)$ or (if $x=z$, then $y \leq w)$. Let $f, g: X \rightarrow X$ be defined by

$$
f(x, y)=(\max \{x, y\}, \min \{x, y\})
$$

and

$$
g(x, y)=\left(\max \{x, y\}, \frac{x+y}{2}\right),
$$

then $f$ and $g$ are weakly increasing mappings. However, both $f$ and $g$ are not nondecreasing.

Example 2 ([4]). Let $X=[1, \infty) \times[1, \infty)$ be endowed with coordinatwise ordering, that is, $(x, y) \preceq(z, w) \Leftrightarrow x \leq z$ and $y \leq w$. Let $f, g: X \rightarrow X$ be defined by $f(x, y)=(2 x, 3 y)$ and $g(x, y)=\left(x^{2}, y^{2}\right)$, then $f(x, y)=(2 x, 3 y) \preceq g f(x, y)=$ $g(2 x, 3 y)=\left(4 x^{2}, 9 y^{2}\right)$ and $g(x, y)=\left(x^{2}, y^{2}\right) \preceq f g(x, y)=f\left(x^{2}, y^{2}\right)=\left(2 x^{2}, 3 y^{2}\right)$. Thus $f$ and $g$ are weakly increasing mappings.

\section{MAIN RESULTS}

Now we present our main theorem for single valued mappings.

Theorem 3. Let $(X, \vartheta)$ be a Hausdorff uniform space and $p$ be an E-distance on $X$ such that $X$ is $S$-complete and let $\phi: X \rightarrow \mathbb{R}$ be a function which is bounded below. If $\preceq$ is the partial order induced by $\phi$ and $f, g: X \rightarrow X$ are two $p$-continuous or $\tau(\vartheta)$-continuous weakly increasing functions, then $f$ and $g$ have a common fixed point in $X$.

Proof. Let $x_{0}$ be an arbitrary point of $X$ and define a sequence $\left\{x_{n}\right\}$ in $X$ as follows:

$$
x_{2 n+1}=f x_{2 n} \text { and } x_{2 n+2}=g x_{2 n+1} \text { for } n \in\{0,1, \cdots\} .
$$

Note that, since $f$ and $g$ are weakly increasing, we have

$$
\begin{aligned}
& x_{1}=f x_{0} \preceq g f x_{0}=g x_{1}=x_{2}, \\
& x_{2}=g x_{1} \preceq f g x_{1}=f x_{2}=x_{3}
\end{aligned}
$$

and by continuing this process we have

$$
x_{1} \preceq x_{2} \preceq \ldots \preceq x_{n} \preceq x_{n+1} \preceq \ldots
$$

So the sequence $\left\{x_{n}\right\}$ is non-decreasing. By the definition of $\preceq$ we have $\cdots \phi\left(x_{2}\right) \leq$ $\phi\left(x_{1}\right) \leq \phi\left(x_{0}\right)$, that is, the sequence $\left\{\phi\left(x_{n}\right)\right\}$ is a non-increasing sequence in $\mathbb{R}$. Since $\phi$ is bounded from below, $\left\{\phi\left(x_{n}\right)\right\}$ is convergent and hence it is Cauchy, i.e. for all $\varepsilon>0$, there exists $n_{0} \in \mathbb{N}$ such that for all $m>n>n_{0}$ we have $\left|\phi\left(x_{m}\right)-\phi\left(x_{n}\right)\right|<$ $\varepsilon$. Since $x_{n} \preceq x_{m}$, we have $x_{n}=x_{m}$ or $p\left(x_{n}, x_{m}\right) \leq \phi\left(x_{n}\right)-\phi\left(x_{m}\right)$. Therefore, 


$$
\begin{aligned}
p\left(x_{n}, x_{m}\right) & \leq \phi\left(x_{n}\right)-\phi\left(x_{m}\right) \\
& =\left|\phi\left(x_{m}\right)-\phi\left(x_{n}\right)\right| \\
& <\varepsilon .
\end{aligned}
$$

which shows that (in view of Lemma 1 (c)) $\left\{x_{n}\right\}$ is $p$-Cauchy sequence. By the $S$ completeness of $X$, there is $z \in X$ such that $\lim _{n \rightarrow \infty} p\left(x_{n}, z\right)=0$. Consequently, by the $p$-continuity of $f$ and $g$, we have $\lim _{n \rightarrow \infty} p\left(f x_{n}, f z\right)=0=\lim _{n \rightarrow \infty} p\left(g x_{n}, g z\right)$. Therefore $\lim _{n \rightarrow \infty} p\left(x_{n}, z\right)=0=\lim _{n \rightarrow \infty} p\left(f x_{n}, f z\right)=\lim _{n \rightarrow \infty} p\left(g x_{n}, g z\right)$ and so from Lemma 1 (a), we have $f z=g z=z$. The proof is similar when $f$ and $g$ are $\tau(\vartheta)$ continuous, since $S$-completeness implies $p$-Cauchy completeness (Remark 1).

In the following we provide two multivalued versions of the preceding theorem. These results are related to the theorems in [9].

Let $X$ be a topological space and $\preceq$ be a partial order on $X$. Let $2^{X}$ denote the family of all nonempty subsets of $X$.

Definition 4 ([9]). Let $A, B$ be two nonempty subsets of $X$, and assume that the relations between $A$ and $B$ are defined as follows:

( $\left.\mathrm{r}_{1}\right)$ If for every $a \in A$, there exists $b \in B$ such that $a \preceq b$, then $A \prec_{1} B$.

$\left(\mathrm{r}_{2}\right)$ If for every $b \in B$, there exists $a \in A$ such that $a \preceq b$, then $A \prec_{2} B$.

( $\left.\mathrm{r}_{3}\right)$ If $A \prec_{1} B$ and $A \prec_{2} B$, then $A \prec B$.

Remark 2 ([9]). $\prec_{1}$ and $\prec_{2}$ are different relations between $A$ and $B$. For example, let $X=\mathbb{R}, A=\left[\frac{1}{2}, 1\right], B=[0,1], \preceq$ be usual order on $X$, then $A \prec_{1} B$ but $A \nprec_{2} B$; if $A=[0,1], B=\left[0, \frac{1}{2}\right]$, then $A \prec_{2} B$ while $A \nprec_{1} B$.

Remark 3 ([9]). $\prec_{1}, \prec_{2}$ and $\prec$ are reflexive and transitive, but are not antisymmetric. For instance, let $X=\mathbb{R}, A=[0,3], B=[0,1] \cup[2,3], \preceq$ be the usual order on $X$, then $A \prec B$ and $B \prec A$, but $A \neq B$. Hence, they are not partial orders on $2^{X}$.

Definition 5 ([9]). A multi-valued operator $T: X \rightarrow 2^{X}$ is called order closed if for monotone sequences $\left\{u_{n}\right\},\left\{v_{n}\right\} \subset X, u_{n} \rightarrow u_{0}, v_{n} \rightarrow v_{0}$ and $v_{n} \in T u_{n}$ imply $v_{0} \in T u_{0}$.

The order closed operators on uniform space using $E$-distance function has been characterised in [3] in the following way.

Definition $6([3])$. Let $(X, \vartheta)$ be a Hausdorff uniform space and let $p$ be an $E$ distance on $X$. A multi-valued operator $T: X \rightarrow 2^{X}$ is called $p$-order closed if for monotone sequences $\left\{u_{n}\right\},\left\{v_{n}\right\} \subset X, \lim _{n \rightarrow \infty} p\left(u_{n}, u_{0}\right)=0, \lim _{n \rightarrow \infty} p\left(v_{n}, v_{0}\right)=0$ and $v_{n} \in T u_{n}$ imply $v_{0} \in T u_{0}$.

Now, we introduce the following definition. 
Definition 7. Let $(X, \preceq)$ be a partially ordered set. Two mappings $F, G: X \rightarrow 2^{X}$ are said to be weakly increasing with respect to $\prec_{1}$ if for any $x \in X$ we have $F x$ $\prec_{1} G y$ for all $y \in F x$ and $G x \prec_{1} F y$ for all $y \in G x$. Similarly two maps $F, G$ : $X \rightarrow 2^{X}$ are said to be weakly increasing with respect to $\prec_{2}$ if for any $x \in X$ we have $G y \prec_{2} F x$ for all $y \in F x$ and $F y \prec_{2} G x$ for all $y \in G x$.

Example 3. Let $X=[1, \infty)$ and $\leq$ be usual order on $X$. Consider two mappings $F, G: X \rightarrow 2^{X}$ defined by $F x=\left[1, x^{2}\right]$ and $G x=[1,2 x]$ for all $x \in X$. Then the pair of mappings $F$ and $G$ are weakly increasing with respect to $\prec_{2}$ but not w.r.t $\prec_{1}$. Indeed, since

$$
G y=[1,2 y] \prec_{2}\left[1, x^{2}\right]=F x \text { for all } y \in F x
$$

and

$$
F y=\left[1, y^{2}\right] \prec_{2}[1,2 x]=G x \text { for all } y \in G x
$$

so $F$ and $G$ are weakly increasing with respect to $\prec_{2}$ but $F 2=[1,4] \nprec_{1}[1,2]=G 1$ for $1 \in F 2$, so $F$ and $G$ are not weakly increasing with respect to $\prec_{1}$.

Example 4. Let $X=[0,1]$ and $\leq$ be usual order on $X$. Consider two mappings $F, G: X \rightarrow 2^{X}$ defined by $F x=\{0,1\}$ and $G x=[x, 1]$ for all $x \in X$. Then the pair of mappings $F$ and $G$ are weakly increasing with respect to $\prec_{1}$ but not w.r.t. $\prec_{2}$. Indeed, since

$$
F x=\{0,1\} \prec_{1}[y, 1]=G y \text { for all } y \in F x
$$

and

$$
G x=[x, 1] \prec_{1}\{0,1\}=F y \text { for all } y \in G x
$$

so $F$ and $G$ are weakly increasing with respect to $\prec_{1}$ but $G 1=\{1\} \nprec_{2}\{0,1\}=F 1$ for $1 \in F 1$, so $F$ and $G$ are are not weakly increasing with respect to $\prec_{2}$.

Two multivalued versions of preceding theorem are as follows:

Theorem 4. Let $(X, \vartheta)$ be a Hausdorff uniform space and $p$ let be an E-distance on $X$ such that $X$ is $S$-complete and $\phi: X \rightarrow \mathbb{R}$ is a function which is bounded from below. If $\preceq$ is the partial order induced by $\phi$ and $F, G: X \rightarrow 2^{X}$ are two order closed and weakly increasing mappings with respect to $\prec_{1}$, then $F$ and $G$ have a common fixed point in $X$.

Proof. Let $x_{0} \in X$ be an arbitrary point. Since $F x_{0} \neq \varnothing$, we can choose $x_{1} \in$ $F x_{0}$. Now since $F$ and $G$ are weakly increasing with respect to $\prec_{1}$, we have $x_{1} \in$ $F x_{0} \prec_{1} G x_{1}$. Thus there exist some $x_{2} \in G x_{1}$ such that $x_{1} \preceq x_{2}$. Again since $F$ and $G$ are weakly increasing with respect to $\prec_{1}$, we have $x_{2} \in G x_{1} \prec_{1} F x_{2}$. Thus there exist some $x_{3} \in F x_{2}$ such that $x_{2} \preceq x_{3}$. By continuing this process, we will get a nondecreasing sequence $\left\{x_{n}\right\}_{n=1}^{\infty}$ which satisfies $x_{2 n+1} \in F x_{2 n}, x_{2 n+2} \in$ $G x_{2 n+1}, n=0,1,2, \ldots$. By the definition of $\preceq$ we have

$$
\cdots \phi\left(x_{3}\right) \leq \phi\left(x_{2}\right) \leq \phi\left(x_{1}\right),
$$


that is, the sequence $\left\{\phi\left(x_{n}\right)\right\}$ is a non-increasing sequence in $\mathbb{R}$. Since $\phi$ is bounded from below, then $\left\{\phi\left(x_{n}\right)\right\}$ is convergent and hence it is Cauchy. That is, for all $\varepsilon>$ 0 , there exists $n_{0} \in \mathbb{N}$ such that for all $m>n>n_{0}$ we have $\left|\phi\left(x_{m}\right)-\phi\left(x_{n}\right)\right|<$ $\varepsilon$. On the other hand, since $x_{n} \preceq x_{m}$, we have $x_{n}=x_{m}$ or $p\left(x_{n}, x_{m}\right) \leq \phi\left(x_{n}\right)-$ $\phi\left(x_{m}\right)$. Therefore, for all $m>n>n_{0}$ we have $p\left(x_{n}, x_{m}\right)<\varepsilon$. This implies $\left\{x_{n}\right\}$ is $p$-Cauchy sequence in $X$. By the $S$-completeness of $X$, there is $z \in X$ such that $\lim _{n \rightarrow \infty} p\left(x_{n}, z\right)=0$. Therefore, $\lim _{n \rightarrow \infty} p\left(x_{2 n+1}, z\right)=0$ and

$\lim _{n \rightarrow \infty} p\left(x_{2 n+2}, z\right)=0$. Consequently, since $F$ and $G$ are $p$-order closed, $\left\{x_{n}\right\}_{n=1}^{\infty}$ monotone and $x_{2 n+1} \in F x_{2 n}, x_{2 n+2} \in G x_{2 n+1}$, we deduce that $z \in F z$ and $z \in G z$, i.e. $z$ is a common fixed point of $F$ and $G$.

Theorem 5. Let $(X, \vartheta)$ be a Hausdorff uniform space and $p$ be an E-distance on $X$ such that $X$ is $S$-complete and $\phi: X \rightarrow \mathbb{R}$ be a function which is bounded below. If $\preceq$ is the partial order induced by $\phi$ and $F, G: X \rightarrow 2^{X}$ are two order closed and weakly increasing mappings with respect to $\prec_{2}$, then $F$ and $G$ have a common fixed point in $X$.

Proof. Let $x_{0} \in X$ be arbitrary point. Since $F x_{0} \neq \varnothing$, we can choose $x_{1} \in F x_{0}$. Now since $F$ and $G$ are weakly increasing with respect to $\prec_{2}$, we have $G x_{1} \prec_{2} F x_{0}$. Thus there exist some $x_{2} \in G x_{1}$ such that $x_{2} \preceq x_{1}$. Again since $F$ and $G$ are weakly increasing with respect to $\prec_{2}$ we have $F x_{2} \prec_{2} G x_{1}$. Thus there exist some $x_{3} \in F x_{2}$ such that $x_{3} \preceq x_{2}$. If we continue this process, we will get a nonincreasing sequence $\left\{x_{n}\right\}_{n=1}^{\infty}$ which satisfies $x_{2 n+1} \in F x_{2 n}, x_{2 n+2} \in T x_{2 n+1}, n=0,1,2, \ldots$ By the definition $\preceq$, we have

$$
\varphi\left(x_{1}\right) \leq \varphi\left(x_{2}\right) \leq \varphi\left(x_{3}\right) \leq \cdots .
$$

Since $\phi$ is bounded from above, then $\left\{\phi\left(x_{n}\right)\right\}$ is convergent and hence it is Cauchy. That is, for all $\varepsilon>0$, there exists $n_{0} \in \mathbb{N}$ such that for all $m>n>n_{0}$ we have $\left|\phi\left(x_{m}\right)-\phi\left(x_{n}\right)\right|<\varepsilon$. On the other hand, since $x_{n} \preceq x_{m}$, we have $x_{n}=x_{m}$ or $p\left(x_{n}, x_{m}\right) \leq \phi\left(x_{n}\right)-\phi\left(x_{m}\right)$. Therefore, for all $m>n>n_{0}$ we have $p\left(x_{n}, x_{m}\right)<\varepsilon$. This implies that $\left\{x_{n}\right\}$ is $p$-Cauchy sequence in $X$. By the $S$-completeness of $X$, there is an $z \in X$ such that $\lim _{n \rightarrow \infty} p\left(x_{n}, z\right)=0$. Therefore, $\lim _{n \rightarrow \infty} p\left(x_{2 n+1}, z\right)=$ 0 and $\lim _{n \rightarrow \infty} p\left(x_{2 n+2}, z\right)=0$. Consequently, since $F$ and $G$ are $p$-order closed, $\left\{x_{n}\right\}_{n=1}^{\infty}$ is monotone and $x_{2 n+1} \in F x_{2 n}, x_{2 n+2} \in G x_{2 n+1}$, we can deduce that $z \in F z$ and $z \in G z$, i.e. $z$ is a common fixed point of $F$ and $G$.

\section{ACKNOWLEDGEMENT}

The author thank the referees for their valuable comments and suggestions.

\section{REFERENCES}

[1] M. Aamri, S. Bennani, and D. El Moutawakil, "Fixed points and variational principle in uniform spaces," Sib. Èlektron. Mat. Izv., vol. 3, pp. 137-142, 2006. 
[2] M. Aamri and D. El Moutawakil, "Common fixed point theorems for $e$-contractive or $e$-expansive maps in uniform spaces," Acta Math. Acad. Paedagog. Nyhazi. (N.S.), vol. 20, no. 1, pp. 83-91, 2004.

[3] I. Altun and M. Imdad, "Some fixed point theorems on ordered uniform spaces," Filomat, vol. 23, no. 3, pp. 15-22, 2009.

[4] I. Altun and H. Simsek, "Some fixed point theorems on ordered metric spaces and application," Fixed Point Theory Appl., vol. 2010, p. 17, 2010.

[5] N. Bourbaki, "Eléments de mathématique. fasc. II." in Livre III: Topologie générale. Chapitre 1: Structures topologiques. Chapitre 2: Structures uniformes., ser. Quatrième édition. Actualités Scientifiques et Industrielles. No. 1142. Paris: Hermann, 1965, p. 225.

[6] A. Brøndsted, “On a lemma of Bishop and Phelps," Pacific J. Math., vol. 55, pp. 335-341, 1974.

[7] B. C. Dhage, "Condensing mappings and applications to existence theorems for common solution of differential equations," Bull. Korean Math. Soc., vol. 36, no. 3, pp. 565-578, 1999.

[8] B. C. Dhage, D. O'Regan, and R. P. Agarwal, "Common fixed point theorems for a pair of countably condensing mappings in ordered Banach spaces," J. Appl. Math. Stochastic Anal., vol. 16, no. 3, pp. 243-248, 2003.

[9] Y. Feng and S. Liu, "Fixed point theorems for multi-valued increasing operators in partial ordered spaces," Soochow J. Math., vol. 30, no. 4, pp. 461-469, 2004.

Author's address

\section{Ishak Altun}

Department of Mathematics, Faculty of Science and Arts, Kirikkale University, 71450-Yahsihan, Kirikkale, Turkey

E-mail address: ishakaltun@yahoo.com 